\title{
Multimorbilidade em Medicina Geral e Familiar: Construção e Validação do Questionário de Avaliação da Sobrecarga da Gestão da Multimorbilidade em Medicina Geral e Familiar (SoGeMM-MGF)
}

\section{Multimorbidity in General and Family Medicine: Construction and Validation of the Questionnaire of Evaluation of Burden of Management of Multimorbidity in General and Family Medicine}

Filipe Prazeres $\# 1,2,3$, Luiz Miguel Santiago \#4,5,6,7, Pedro Miguel Pereira4 , Pedro Miguel Santos ${ }^{4}$, Telmo Cortinhal ${ }^{4}$

Autor Correspondente/Corresponding Author:

Filipe Prazeres [filipeprazeresmd@gmail.com] Rua Padre Rubens, 3830-596 Gafanha da Nazaré, Portugal

ORCID iD: 0000-0002-2849-5194

\section{RESUMO}

INTRODUÇÃO: A gestão da multimorbilidade pode originar sobrecarga médica que deve ser avaliada.

O objetivo do trabalho foi criar e validar um questionário para medir a sobrecarga pela gestão da multimorbilidade nos médicos de Medicina Geral e Familiar.

MÉTODOS: O questionário foi construído após revisão da literatura e das informações de um grupo focal. Validade de constructo efetuada pelo método da máxima verossimilhança para análise fatorial e rotação oblíqua simples (oblimin), numa amostra de médicos de Medicina Geral e Familiar após pré-teste.

RESULTADOS: Validação com estudo das respostas de 223 médicos de Medicina Geral e Familiar (120 mulheres), média etária de 51,4 \pm 13,1 anos, do centro de Portugal, via convite a preenchimento do questionário online. Após análise o questionário foi reduzido de 23 para 16 itens demonstrando boa coerência interna e validade de constructo, com quatro fatores explicando 66,6\% da variância total do questionário. Alfa de Cronbach, para a escala global de 0,89 e fatores entre $0,88 X$ e $0,49 Y$.

\#Os autores contribuíram de igual forma para a elaboração do presente trabalho/ \#Joint first authors

1. Faculdade de Ciências da Saúde, Universidade da Beira Interior, Covilhã, Portugal. 2. USF Beira Ria, Gafanha da Nazaré, Portugal. 3. Centre for Health Technology and Services Research (CINTESIS), Porto, Portugal. 4. Clínica Universitária de Medicina Geral e Familiar da Faculdade de Medicina da Universidade de Coimbra, Coimbra, Portugal. 5. CEISUC - Centro de Estudos de Investigação em Saúde da Universidade de Coimbra, Coimbra, Portugal. 6. Centre for Health Studies and Research of the University of Coimbra, Coimbra, Portugal. 7. University Clinic of General Practice and Family Medicine.

• Autor (es) (ou seu (s) empregador (es)) 2019. Reutilização permitida de acordo com CC BY-NC. Nenhuma reutilização comercial. ${ }^{\circledR}$ Author(s) (or their employer(s)) 2019. Re-use permitted under CC BY-NC. No commercial re-use. 
DISCUSSÃO: SoGeMM-MGF de 16 questões permite avaliar quatro áreas de impacto da multimorbilidade. Este instrumento esclarece áreas e dificuldades sentidas pelos profissionais médicos, e poderá orientar o desenvolvimento de soluções para uma gestão mais eficaz da multimorbilidade. Apesar das boas propriedades psicométricas do questionário, estudos mais robustos da sua validade concorrente e discriminativa e estabilidade temporal, em amostras mais numerosas e em outras zonas do país, serão necessários futuramente.

CONCLUSÃO: O questionário pode ser considerado um instrumento fiável para a avaliação da sobrecarga da gestão da multimorbilidade em Medicina Geral e Familiar.

PALAVRAS-CHAVE: Cuidados Centrados no Doente; Gestão da Doença; Inquéritos e Questionários; Medicina Geral e Familiar; Médicos de Família; Multimorbilidade

\section{ABSTRACT}

INTRODUCTION: The management of multimorbidity can lead to medical burden that should be evaluated.

Our objective was to create and validate a questionnaire to measure the burden of the management of multimorbidity in Family Physicians.

MATERIAL AND METHODS: The questionnaire was constructed after a literature review on multimorbidity and information from a focus group. The construct validity was performed using the maximum likelihood method for factor analysis, followed by simple oblique rotation (oblimin), in a sample of family physicians after a pre-test.

RESULTS: Validation of the study of 223 family physicians' responses (120 women), mean age of $51.4 \pm 13.1$ years, from the centre of Portugal, through an invitation to complete the online questionnaire. After the analysis, the questionnaire was reduced from 23 to 16 items, showing good internal consistency and construct validity, with four factors explaining 66.6\% of the total variance of the questionnaire. Cronbach's alpha, for the global scale of 0.89 and factors between 0.88X and 0.49Y. DISCUSSION: SoGeMM-MGF with 16 questions allows to evaluate 4 areas of impact of multimorbidity. This tool clarifies areas and difficulties experienced by medical professionals and may guide the development of solutions for a more effective management of multimorbidity. Despite the good psychometric properties of the questionnaire, in the future more robust studies of its concurrent and discriminative validity and temporal stability in larger samples and in other areas of the country will be necessary.

CONCLUSION: The questionnaire can be considered a reliable instrument for assessing the burden of multimorbidity management in general and family medicine.

KEYWORDS: Disease Management; Family Practice; General Practitioners; Multimorbidity; Patient-Centered Care; Surveys and Questionnaires

\section{INTRODUÇÃO}

A multimorbilidade (MM), tem constructo ainda não consensualmente definido, sendo a sua definição europeia, traduzida em múltiplas línguas ${ }^{1}$ e cientificamente validada em publicações internacionais, ${ }^{2}$ descrita holisticamente, para os cuidados de saúde primários como "... qualquer combinação de uma doença crónica com pelo menos uma outra doença (aguda ou crónica), ou com um fator biopsicossocial (associado ou não), ou com um fator de risco somático."3

A MM representa um desafio atual, afetando cerca de 50 milhões de pessoas na Europa. ${ }^{4}$

A MM é também um problema de saúde comum nos adultos portugueses que frequentam as consultas de Medicina Geral e Familiar (MGF), sendo mais prevalen- te nos doentes idosos. ${ }^{5}$ Tendo em conta o envelhecimento das populações e o aumento da esperança média de vida, ${ }^{6}$ o número de doentes com este problema de saúde aumentará no futuro.

A MM está associada a maior utilização de cuidados de saúde, aumento do custo associado ao tratamento, ${ }^{7}$ pior qualidade de vida, ${ }^{8}$ período de internamento mais prolongado, ${ }^{9}$ aumento da incapacidade ou fragilidade, ${ }^{3} \mathrm{e}$ aumento da mortalidade. ${ }^{9}$

A investigação científica sobre a perspetiva médica da gestão da multimorbilidade encontra-se ainda numa fase precoce. Os estudos pioneiros nesta temática integraram procedimentos de natureza qualitativa, fazendo uso de grupos focais compostos por médicos que consideram que a gestão destes doentes é exigente, complexa e associada a maior carga de trabalho. ${ }^{10-12}$ 
Existe assim a necessidade de um instrumento que permita ao próprio médico e aos decisores em saúde perceber como a gestão da MM está ou não a afetar o bem-estar do médico, fator fundamental para o ótimo desempenho terapêutico; podendo aquele ser considerado um indicador de qualidade. ${ }^{13}$

Pelas pesquisas efetuadas não existe um instrumento validado em Portugal (ou mesmo internacionalmente) para avaliar de que forma é que os médicos de Medicina Geral e Familiar sentem a sobrecarga da gestão da multimorbilidade.

A construção de um questionário com características qualitativas e quantitativas permitirá identificar as áreas de maior dificuldade na gestão dos doentes complexos, e dentro das mesmas, quantificar a sobrecarga que esta representa para o médico de Medicina Geral e Familiar.

O objetivo do presente trabalho é a construção e validação de um instrumento de avaliação da sobrecarga da gestão da multimorbilidade em Medicina Geral e Familiar.

\section{MATERIAL E MÉTODOS}

Tendo em conta o objetivo da presente investigação, desenvolvemos um estudo metodológico e transversal. Procedeu-se à construção do Questionário de Avaliação da Sobrecarga da Gestão da Multimorbilidade em MGF (SoGeMM-MGF). À semelhança de estudos anteriores nos quais o formato do instrumento foi inspirado em questionários previamente existentes,,$^{14}$ o SoGeMM-MGF adotou o formato quantitativo do instrumento de Bandeira et al. ${ }^{15}$ Não obstante, as áreas de sobrecarga a avaliar não correspondem às identificadas por Bandeira et $a^{15}$ pois foram incluídas as anteriormente identificadas no estudo de Prazeres \& Santiago, ${ }^{10}$ bem como na revisão da literatura. Posteriormente, o questionário foi submetido à avaliação por consenso através de um focus group realizado com 10 médicos de Medicina Geral e Familiar (6 médicos especialistas e 4 médicos internos) em fevereiro de 2018. A participação foi voluntária e teve a duração de cerca de 2 horas. Não existiu a necessidade de eliminar questões. Foram acrescentadas 4 questões consideradas importantes ao contexto prático do médico de família que lida com doentes com multimorbilidade.

O questionário inicial foi constituído por 23 questões de autopreenchimento de escalas tipo Likert com 5 alternativas de resposta. A chave da cotação variava entre 1 , 2, 3, 4 e 5. Quanto maior a cotação atribuída a um item, maior a sobrecarga associada ao mesmo.

Foi também utilizado um conjunto de questões para caracterização dos médicos inquiridos: idade; sexo; estado civil; anos de trabalho com doentes com multimorbilidade; local de trabalho; familiaridade do termo multimorbilidade; frequência de curso específico sobre multimorbilidade; número médio de consultas que realiza por semana; número médio de consultas realizadas por semana a doentes com multimorbilidade; proporção de doentes com multimorbilidade no ficheiro médico.

Realizou-se um pré-teste em março de 2018. O questionário de 23 questões foi aplicado a um conjunto de 32 médicos especialistas em MGF, no seu local de trabalho. Estes responderam ao questionário em dois tempos, num formato teste e re-teste, sendo a primeira vez de forma escrita pelos próprios e subsequentemente por via oral. O intervalo entre aplicações do questionário foi sempre de pelo menos um dia.

O convite para a participação no estudo foi enviado pela Secção Regional do Centro da Ordem dos Médicos em setembro de 2018, por correio eletrónico, para 511 médicos especialistas e internos de formação específica em Medicina Geral e Familiar. Os participantes acederam a um link informático para o servidor onde se encontrava o questionário, sendo a recolha das respostas automática após o preenchimento e submissão, considerando-se consentimento informado a resposta obtida. Foi garantido o anonimato, confidencialidade e sigilo dos participantes.

Os resultados do presente estudo foram analisados através do IBM SPSS Statistics for Windows, V.21,0 (IBM Corporation, Armonk, New York, USA). Após a construção do questionário, a validação psicométrica foi realizada através da validade de construto e consistência interna. Definiu-se como estatisticamente significativo um valor de $p<0,05$.

O trabalho foi aprovado pela Comissão de Ética da Administração Regional de Saúde (ARS) Centro e realizado de acordo com a declaração de Helsínquia.

\section{RESULTADOS}

\section{TESTE E RE-TESTE}

$\mathrm{Na}$ fase de pré-teste estiveram envolvidos 32 médicos de MGF da Administração Regional de Saúde (ARS) do Centro de Portugal, com idade média de 48,22 \pm 14,54 anos, sendo 11 (34,4\%) do sexo masculino, trabalhando, em Unidade de Saúde Familiar (USF) (68,7\%), particularmente de modelo B (modelo em "pay for performance" (P4P)) (53,1\%) e 31,3\% em Unidades de Cuidados de Saúde Personalizados (UCSP). A confiabilidade foi de 0,510 . 
A correlação positiva e significativa $(\rho=0,854 ; p<0,001)$ obtida nas duas aplicações espaçadas em média de 3 semanas, não indicou diferenças entre o teste e o re-teste.

\section{AMOSTRA DA FASE DE ESTUDO DE CAMPO}

A informação demográfica e profissional relativa aos participantes é apresentada na Tabela 1. A amostra consistiu em 223 médicos de família (120 do sexo feminino), com idades compreendidas entre 25 e 70 anos (Média $=51,4 \pm 13,1$ anos). A maioria referiu familiaridade com o termo multimorbilidade (89,2\%), trabalhar há mais de 10 anos com doentes com multimorbilidade (65,9\%) e realizar trinta ou mais consultas por semana a estes doentes (84,3\%) (Tabela 1).

\section{PROPRIEDADES PSICOMÉTRICAS DO QUESTIONÁRIO SoGeMM-MGF}

Efetuou-se uma primeira análise de correlação simples entre os itens do questionário. O item 21 - "o sistema de recolha e tratamento de informação que usa na consulta facilita a gestão do doente com multimorbilidade?" - correlacionou-se fracamente $(r<0,3)$ com os outros itens e consequentemente foi removido. Os restantes itens apresentavam correlações significativas.

Posteriormente, a validade de construto do questionário com 22 itens foi realizada através do método da máxima verossimilhança para análise fatorial, ${ }^{16}$ seguida de rotação oblíqua simples (oblimin), sendo definidos como critérios de extração de fatores: eigenvalues > 1,0 (critério de Kaiser-Guttman) e na observação do gráfico dos eigenvalues (teste scree plot); e para retenção de itens: coeficientes de saturação > 0,32. ${ }^{17} \mathrm{Na}$ sequência destes procedimentos foram eliminados 6 itens adicionais ("4. Quão frequentemente se sente o(a) responsável por todo o cuidado necessário aos doentes com multimorbilidade?"; "15.De uma forma geral, sente que os doentes com multimorbilidade hipervalorizam o seu estado de saúde e desvalorizam a sua terapêutica?"; "16.Em muitos aspetos, o seu trabalho com doentes com multimorbilidade aproxima-se dos seus ideais da prática médica?"; "19.Se pudesse alterar o seu processo de trabaIho para com o doente com multimorbilidade fá-lo-ia?"; "20.Considera que tem falta do equipamento necessário para lidar com doentes com multimorbilidade?" e "23.De uma forma geral, quão satisfatório é para si lidar com doentes com multimorbilidade?"), ficando a versão final do SoGeMM-MGF com 16 itens.

Com a eliminação dos referidos 7 itens a proporção participante/variável foi de cerca de 14:1. O teste de Bartlett apresentou nível de significância inferior a $p<$ 0,05 (qui-quadrado [120] = 1703,6 p<0,0001). 0 coe-
TABELA 1. Características demográficas e profissionais dos participantes. $(\mathrm{N}=223)$

\begin{tabular}{|c|c|c|}
\hline & $\mathrm{N}$ & $\%$ \\
\hline \multicolumn{3}{|l|}{ Sexo } \\
\hline Masculino & 66 & 29,6 \\
\hline Feminino & 120 & 53,8 \\
\hline Idade (anos) média \pm DP & $51,4 \pm 13,1$ & \\
\hline \multicolumn{3}{|l|}{ Estado civil } \\
\hline Solteiro & 7 & 3,1 \\
\hline Casado ou vive com companheiro & 21 & 9,4 \\
\hline Separado & 4 & 1,8 \\
\hline \multicolumn{3}{|l|}{ Local de trabalho } \\
\hline UCSP & 70 & 31,4 \\
\hline USF Modelo A & 60 & 26,9 \\
\hline USF Modelo B & 56 & 25,1 \\
\hline \multicolumn{3}{|l|}{ Familiaridade com o termo MM } \\
\hline Sim & 199 & 89,2 \\
\hline Não & 1 & 0,4 \\
\hline \multicolumn{3}{|c|}{ Tempo de trabalho com doentes com MM } \\
\hline$>10$ anos & 147 & 65,9 \\
\hline 6-10 anos & 60 & 26,9 \\
\hline$\leq 5$ anos & 7 & 3,1 \\
\hline \multicolumn{3}{|c|}{ Frequência de curso específico sobre MM } \\
\hline Sim & 25 & 11,2 \\
\hline Não & 192 & 86,1 \\
\hline \multicolumn{3}{|c|}{$N^{\circ}$ médio de consultas realizadas por semana } \\
\hline$<70$ & 21 & 9,4 \\
\hline 70-99 & 58 & 26,0 \\
\hline $100-130$ & 84 & 37,7 \\
\hline$>130$ & 59 & 26,5 \\
\hline \multicolumn{3}{|c|}{$N^{\circ}$ médio de consultas por semana a doentes com MM } \\
\hline$<30$ & 11 & 4,9 \\
\hline $30-59$ & 76 & 34,1 \\
\hline $60-90$ & 62 & 27,8 \\
\hline$>90$ & 50 & 22,4 \\
\hline \multicolumn{3}{|l|}{$\%$ de doentes com MM no ficheiro } \\
\hline$\leq 25 \%$ & 22 & 9,9 \\
\hline$\geq 26$ e $\leq 50 \%$ & 115 & 51,6 \\
\hline$\geq 51 \mathrm{e} \leq 70 \%$ & 65 & 29,1 \\
\hline$\geq 71 \%$ & 19 & 8,5 \\
\hline
\end{tabular}

MM: multimorbilidade; DP: desvio-padrão

Percentagens inferiores a 100\% refletem ausência de resposta a questões demográficas e/ou profissionais

ficiente de Kaiser-Meyer-Olkin (KMO) revelou um valor de 0,85, permitindo análise fatorial subsequente e julgando-se a amostra adequada.

Através do método da máxima verossimilhança para análise fatorial, foram extraídos quatro fatores, explicando 66,6\% da variância total do SoGeMM-MGF, com pesos fatoriais entre 0,92 e 0,35 (Tabela 2). 
TABELA 2. Itens retidos a partir do método da máxima verossimilhança para análise fatorial, seguida de rotação oblíqua.

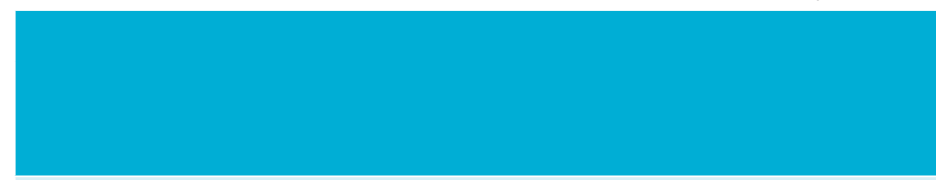

13. Lidar com doentes com multimorbilidade causa-Ihe ansiedade?

9. De uma forma geral, lidar com doentes com multimorbilidade afeta a sua estabilidade emocional?

8. Sente-se cansado fisicamente por lidar frequentemente com doentes com multimorbilidade?

12. Sente-se frustrado(a) por não conseguir melhorar a gestão dos seus doentes com multimorbilidade?

17. Sente que na consulta tem o tempo suficiente para gerir os problemas do seu doente com multimorbilidade?

\section{FATORES}

\begin{tabular}{|c|c|c|c|}
\hline $\begin{array}{c}|c| \\
\text { Sentimentos } \\
\text { negativos }\end{array}$ & $\begin{array}{c}2 \\
\text { Cooperação }\end{array}$ & $\begin{array}{c}3 \\
\text { Trabalho } \\
\text { Clínico }\end{array}$ & $\begin{array}{c}\text { Gestão da } \\
\text { consulta }\end{array}$ \\
\hline 0,922 & & & \\
\hline
\end{tabular}

0,901

0,744

0,644

0,398

22. Dados os problemas apresentados pelo doente com multimorbilidade este deveria ser entendido como tendo o peso de unidade ponderada extra para efeitos de tamanho de lista?

11. O apoio e resposta atempada às suas dúvidas por outras especialidades ajudaria na gestão do doente com multimorbilidade?

3. O apoio de outro profissional (ex. psicólogo, nutricionista, dentista, gerontólogo social) tornaria o seu trabalho de lidar com doentes com multimorbilidade menos sobrecarregado?

10. O seu trabalho com doentes com multimorbilidade é fácil pois segue as orientações de médicos de outras especialidades?

0,882

7. O seu trabalho com doentes com multimorbilidade é fácil pois segue as Normas de Orientação da DGS?

0,618

14. O seu trabalho com doentes com multimorbilidade é fácil ao cumprir os indicadores da ACSS?

6. Considera excelentes as suas condições de trabalho para lidar com doentes com multimorbilidade?

5. Receia iatrogenia quando lida com doentes com multimorbilidade?

1. Lidar com doentes com multimorbilidade reduz o tempo que tem disponível para os outros doentes?

2. Sente-se sobrecarregado(a) pela proporção de doentes com multimorbilidade que vê na sua consulta?

18. Considera que está mal preparado para gerir de forma integrada os doentes com multimorbilidade?

O primeiro fator, Sentimentos Negativos, explicou $39,1 \%$ da variância dos resultados. O segundo fator, Cooperação, explicou 11,6\% da variância dos resultados. O terceiro fator, Trabalho Clínico, explicou 9,2\% da variância dos resultados. E o quarto fator, Gestão da Consulta, explicou 6,7\% da variância dos resultados. Foi obtida uma qualidade de ajustamento aceitável ${ }^{16}$ (NFI=0,91; TLI=0,88; CFI=0,94; RMSEA=0,08), em suporte da validade fatorial do SoGeMM-MGF.

A consistência interna foi avaliada através do cálculo do coeficiente Alfa de Cronbach. ${ }^{18} \mathrm{O}$ Questionário SoGeMM-MGF de 16 itens obteve um Alfa de 0,89, indicando consistência interna quase perfeita. Por fator, os valores Alfa de Cronbach foram de 0,88; 0,85; 0,79 e 0,49 (quase perfeito a moderado) para sentimentos negativos, cooperação, trabalho clínico e gestão da consulta, respetivamente.
Efetuou-se ainda o cálculo das correlações ${ }^{19}$ entre cada um dos fatores do questionário e o valor global do SoGeMM-MGF, observando-se correlações positivas fortes, entre os quatro fatores e o questionário global $(p<0,01)$, sentimentos negativos $(r=0,847)$, cooperação ( $r=0,675)$, trabalho clínico ( $r=0,813)$, e gestão da consulta $(r=0,782)$. Verificou-se, também, a presença de correlações positivas e estatisticamente significativas entre todos os fatores do questionário (Tabela 3).

\section{DISCUSSÃO}

A ausência de um instrumento adequado e já aferido para a avaliação da sobrecarga da gestão da multimorbilidade por parte dos médicos de MGF constituiu o motivo principal para a realização da validação do questionário SoGeMM-MGF, desconhecendo-se a existência de instrumentos com objetivos semelhantes validados 
TABELA 3. Correlação de Pearson entre os fatores e o total do SoGeMM-MGF.

\begin{tabular}{|l|c|c|c|c|}
\hline & $\begin{array}{c}1 \\
\text { Senti- } \\
\text { mentos } \\
\text { negativos }\end{array}$ & $\begin{array}{c}2 \\
\text { Coope- } \\
\text { ração }\end{array}$ & $\begin{array}{c}3 \\
\text { Tra- } \\
\text { balho } \\
\text { Clínico }\end{array}$ & $\begin{array}{c}4 \\
\text { Gestão } \\
\text { da con- } \\
\text { sulta }\end{array}$ \\
\hline $\begin{array}{l}\text { SoGeMM- } \\
\text {-MGF total }\end{array}$ & $0,847^{* *}$ & $0,675^{* *}$ & $0,813^{* *}$ & $0,782^{* *}$ \\
\hline $\begin{array}{l}\text { Sentimentos } \\
\text { negativos }\end{array}$ & 1 & $0,348^{* *}$ & $0,561^{* *}$ & $0,636^{* *}$ \\
\hline $\begin{array}{l}\text { Cooperação } \\
\begin{array}{l}\text { Trabalho } \\
\text { clínico }\end{array}\end{array}$ & 1 & $0,390^{* *}$ & $0,416^{* *}$ \\
\hline $\begin{array}{l}\text { Gestão da } \\
\text { consulta }\end{array}$ & & & 1 & $0,579^{* *}$ \\
\hline $\begin{array}{c}* * \\
p<0,01\end{array}$ & & & 1 \\
\hline
\end{tabular}

para a língua portuguesa.

Verificou-se que o termo multimorbilidade era familiar para a quase totalidade dos médicos de MGF e que a frequência de formação específica no assunto é diminuta, tal como já anteriormente referido. ${ }^{10} \bigcirc$ termo multimorbilidade sendo relativamente novo, ${ }^{20}$ corresponde ao dia-a-dia dos médicos da especialidade de MGF. ${ }^{21}$ Para 63,7\% da amostra há a realização de 70 a 130 consultas semanais, suspeitando os médicos terem em média por semana $61,9 \%$ das consultas em pessoas com multimorbilidade, numa perspetiva de $80,7 \%$ dos médicos de família referirem a presença de multimorbilidade no ficheiro clínico entre 26\% e 70\%.

$O$ presente trabalho permitiu conceber o instrumento SoGeMM-MGF de 16 questões com critérios de validade e confiabilidade mínimos para avaliar quatro áreas de impacto e que podem ajudar a reduzir a sobrecarga que, a prazo, pode originar sofrimento nos médicos. ${ }^{10}$

Este instrumento permite tirar conclusões concretas, esclarecer áreas e dificuldades sentidas pelos profissionais, e orientar assim o desenvolvimento de soluções para uma gestão mais eficaz da multimorbilidade. Apesar de ter sido utilizada uma escala numérica, trata-se mesmo assim de uma avaliação subjetiva.

Apesar das boas propriedades psicométricas do Questionário SoGeMM-MGF, estudos mais robustos da sua validade concorrente e discriminativa e estabilidade temporal, em amostras mais numerosas e em outras zonas do país, serão necessários no futuro.

\section{CONCLUSÃO}

O Questionário SoGeMM-MGF demonstrou boa coerência interna e validade de constructo podendo ser considerado um instrumento fiável para a avaliação da sobrecarga da gestão da multimorbilidade em MGF.
Sentimentos Negativos, Cooperação, Trabalho Clínico e Gestão da Consulta são assim áreas que futuramente devem ser estudadas para a mais adequada intervenção formativa para evitar distúrbio nos cuidados por haver médicos demasiado "sobrecarregados" e que assim acabam por menos valorizar as queixas dos seus concidadãos reduzindo a sua capacidade terapêutica.

CONFLITOS DE INTERESSE: Os autores declaram não ter qualquer conflito de interesse na realização do presente trabalho.

FONTES DE FINANCIAMENTO: Não houve qualquer fonte de financiamento na realização do presente trabalho.

CONFIDENCIALIDADE DOS DADOS: Os autores declaram ter seguido os protocolos da sua instituição acerca da publicação dos dados de doentes.

PROTEÇÃO DE PESSOAS E ANIMAIS: Os autores declaram que os procedimentos seguidos na elaboração do presente trabalho estão em conformidade com as normas das comissões de investigação clínica e de ética, bem como da declaração de Helsínquia e da Associação Médica Mundial.

PROVENIÊNCIA E REVISÃO POR PARES: Não comissionado; revisão externa por pares.

CONFLICTS OF INTEREST: The authors declare that they have no conflicts of interest.

FINANCIAL SUPPORT: This work has not received any contribution, grant or scholarship.

CONFIDENTIALITY OF DATA: The authors declare that they have followed the protocols of their work center on the publication of data from patients.

PROTECTION OF HUMAN AND ANIMAL SUBJECTS: The authors declare that the procedures followed were in accordance with the regulations of the relevant clinical research ethics committee and with those of the Code of Ethics of the World Medical Association (Declaration of Helsinki).

PROVENANCE AND PEER REVIEW: Not commissioned; externally peer reviewed. 


\section{REFERÊNCIAS}

1. Le Reste JY, Nabbe P, Rivet C, Lygidakis C, Doerr C, Czachowski S, et al. The European general practice research network presents the translations of its comprehensive definition of multimorbidity in family medicine in ten European languages. PLoS One. 2015;10:e0115796. doi: 10.1371/journal. pone.0115796.

2. Le Reste JY, Nabbe P, Manceau B, Lygidakis C, Doerr C, Lingner $\mathrm{H}$, et al. The European General Practice Research Network presents a comprehensive definition of multimorbidity in family medicine and long term care, following a systematic review of relevant literature. J Am Med Dir Assoc. 2013;14:31925. doi: 10.1016/j.jamda.2013.01.001.

3. Prazeres F, Santiago LM, Simoes JA. Defining Multimorbidity: From English to Portuguese Using a Delphi Technique. Biomed Res Int. 2015:2015:965025. doi: 10.1155/2015/965025.

4. Rijken M, Struckmann V, Dyakova M, Melchiorre M, Rissanen S, van Ginneken E. ICARE4EU: Improving care for people with multiple chronic conditions in Europe. Eurohealthcare. 2013; 19: 29-31.

5. Prazeres F, Santiago L. Prevalence of multimorbidity in the adult population attending primary care in Portugal: a cross-sectional study. BMJ Open. 2015;5:e009287. doi: 10.1136/ bmjopen-2015-009287.

6. He W, Goodkind D, Kowal P. An Aging World: 2015. Washington: Government Publishing Office; 2016.

7. Glynn LG, Valderas JM, Healy P, Burke E, Newell J, Gillespie $P$, et al. The prevalence of multimorbidity in primary care and its effect on health care utilization and cost. Fam Pract. 2011;28:516-23. doi: 10.1093/fampra/cmr013.

8. Prazeres F, Santiago L. Relationship between health-related quality of life, perceived family support and unmet health needs in adult patients with multimorbidity attending primary care in Portugal: a multicentre cross-sectional study. Health Qual Life Outcomes. 2016;14:156.

9. Robertson L, Ayansina D, Marks A, Black C. Comparing two measures of multimorbidity in hospitalised patients. Int J Popul Data Sci. 2018; 3:2

10. Prazeres F, Santiago L. The knowledge, awareness, and practices of portuguese general practitioners regarding multimorbidity and its management: qualitative perspectives from open-ended questions. Int J Environ Res Public Health. 2016;13: pii: E1097.

11. Sinnott C, Mc Hugh S, Browne J, Bradley C. GPs' perspectives on the management of patients with multimorbidity: systematic review and synthesis of qualitative research. BMJ Open. 2013;3:e003610. doi: 10.1136/bmjopen-2013-003610.

12. Moth G, Vestergaard M, Vedsted P. Chronic care management in Danish general practice--a cross-sectional study of workload and multimorbidity. BMC Fam Pract. 2012;13:52. doi: 10.1186/1471-2296-13-52.

13. Wallace JE, Lemaire JB, Ghali WA. Physician wellness: a missing quality indicator. Lancet. 2009:374:1714-21. doi: 10.1016/S0140-6736(09)61424-0.

14. Amorim M, Silva I. Instrument for Assessing Critical Thinking in Students and Health Professionals. Psicol Saúde Doenças. 2014; 15: 122-37.

15. Bandeira M, Ishara S, Zuardi AW. Satisfação e sobrecarga de profissionais de saúde mental: validade de construto das escalas SATIS-BR e IMPACTO-BR. J Brasil Psiquiatr. 2007; 56: 280-6.

16. Gignac GE. Partial confirmatory factor analysis: described and illustrated on the NEO-PI-R. J Pers Assess. 2009;91:40-7. doi: 10.1080/00223890802484126.
17. Costello AB, Osborne JW. Best practices in exploratory factor analysis: four recommendations for getting the most from your analysis. Pract Assess Res Eval. 2005; 10:7.

18. Landis JR, Koch GG. The measurement of observer agreement for categorical data. Biometrics. 1977; 33: 159-74.

19. Evans JD. Straightforward Statistics for the Behavioral Sciences. Pacific Grove: Brooks/Cole Publishing; 1996.

20. Santiago LM. Multimorbilidade e Diabetes. Rev Factores Risco. 2016; 36: 58-61.

21. Fortin M, Bravo G, Hudon C, et al. Relationship between multimorbidity and health-related quality of life of patients in primary care. Health Qual Life Outcomes. 2016;14:156. 